\title{
NÍVEIS CRÍTICOS DE BORO EM SOLOS DE VÁRZEA PARA O CULTIVO DO FEIJOEIRO'
}

\author{
EDUARDO DAL'AVA MARIANO², VALDEMAR FAQUIN³, ANTONIO EDUARDO FURTINI NETO ${ }^{4}$ \\ ALEX TEIXEIRA ANDRADE ${ }^{5}$ E ISABELA ORLANDO DOS SANTOS MARIANO 6
}

\begin{abstract}
RESUMO - O experimento foi conduzido em casa de vegetação, com o objetivo de estimar os níveis críticos inferiores (equivalentes a $90 \%$ da produção máxima de grãos) e superiores (equivalentes à redução de $10 \%$ na produção máxima de grãos) de B nos solos e nas plantas de feijoeiro (Phaseolus vulgaris $\mathrm{L}$.) cultivado em amostras de solos de várzea. $\mathrm{O}$ delineamento foi inteiramente casualizado, com quatro repetições, arranjado num esquema fatorial 4 x 7, sendo quatro solos (Glei Pouco Húmico, Aluvial, Glei Húmico e Orgânico) e sete doses de B (0,0, 0,25, 0,5, 1,5, 3,0, 6,0 e 10,0 mg dm d. $^{-3}$. As amostras dos solos, incubadas por 24 dias com calcário dolomítico, macro e micronutrientes e $\mathrm{B}$, foram analisadas no tocante a $\mathrm{B}$ pelos extratores $\mathrm{BaCl}_{2} 0,125 \%$, Mehlich I, água quente e $\mathrm{CaCl}_{2}$ 0,01 mol L-1. Três plantas foram cultivadas por vaso de $3 \mathrm{dm}^{3}$ : uma, colhida no florescimento, avaliando-se os teores foliares de B, e duas, na maturação de grãos, avaliando-se a matéria seca de grãos. Os resultados mostraram que nos solos, os níveis críticos inferiores de B variaram de 0,57 a 1,87 $\mathrm{mg} \mathrm{dm}^{-3}$, e os superiores, de 1,89 a 4,65 mg dm${ }^{-3}$, independentemente do extrator utilizado. Nas folhas do feijoeiro, os níveis críticos inferiores estiveram entre 44,2 e $68,1 \mathrm{mg} \mathrm{kg}^{-1}$, e os superiores, entre 143,6 e 199,1 mg kg-1.
\end{abstract}

Termos para indexação: deficiências do solo, carências minerais, micronutrientes, extratores.

\section{CRITICAL LEVELS OF BORON IN LOWLAND SOILS FOR CULTURE OF BEAN}

\begin{abstract}
This experiment was carried out under greenhouse conditions with the objective of determining the inferior (equivalent to $90 \%$ of the maximum grain yield) and the superior critical levels (equivalent to decrease of $10 \%$ of the maximum grain yield) of B in the soil as well as in the top of common bean plants (Phaseolus vulgaris L.) cultivated in lowland soils samples. The experimental design was completely randomized, with four replications, in a factorial scheme, using four types of soils (Low Humic Gley, Alluvial, Humic Gley and Bog Soil) and seven B doses (0.0, 0.25, 0.50, 1.50, $3.0,6.0$ and $10.0 \mathrm{mg} \mathrm{dm}^{-3}$ ). The soil samples were incubated with lime, macro and micronutrients including B, during 24 days. The soil samples were then submitted to B extraction with four extractant solutions: $\mathrm{BaCl}_{2} 0.125 \%$, Mehlich I, hot water, and $0.01 \mathrm{~mol} \mathrm{~L}^{-1} \mathrm{CaCl}_{2}$. Three plants were cultivated in pots containing three $\mathrm{dm}^{3}$ of treated soil. Boron contents of plant shoots at the flowering period and grain dry matter yield at the end of the cycle were determined. In soils, the inferior critical levels varied from 0.57 to $1.87 \mathrm{mg} \mathrm{dm}^{-3}$ and the superior ones varied from 1.89 to $4.65 \mathrm{mg} \mathrm{dm}^{-3}$. In plants, the inferior critical levels varied from 44.2 to $68.1 \mathrm{mg} \mathrm{kg}^{-1}$ and the superior ones from 143.6 to $199.1 \mathrm{mg} \mathrm{kg}^{-1}$.
\end{abstract}

Index terms: soil deficiencies, mineral deficiencies, trace elements, extractors.

\footnotetext{
${ }^{1}$ Aceito para publicação em 2 dezembro de 1999.

Extraído da dissertação de mestrado apresentada pelo primeiro autor à Universidade Federal de Lavras (UFLA).

Trabalho financiado pelo CNPq e FAPEMIG.

${ }^{2}$ Eng. Agrôn., M.Sc., aluno do curso de doutorado, Wageningen Agricultural University, Holanda. E-mail: Eduardo.Mariano@BodVru.BenP.WAU.NL

${ }^{3}$ Eng. Agrôn., Dr., Prof. Titular, Dep. de Ciência do Solo,
}

UFLA, Caixa Postal 37, CEP 37200-000 Lavras, MG. Bolsista do CNPq. E-mail: vafaquin@ufla.br

${ }^{4}$ Eng. Agrôn., Dr., Prof. Adjunto, Dep. de Ciência do Solo, UFLA. Bolsista do CNPq. E-mail: afurtini@ufla.br

${ }^{5}$ Eng. Agrôn., aluno do curso de mestrado, UFLA. Bolsista da CAPES. E-mail: alex@ufla.br

${ }^{6}$ Eng. Agrôn., aluna do curso de mestrado, Wageningen Agricultural University, Holanda.

E-mail: iosam@hotmail.com 


\section{INTRODUÇÃO}

O cultivo do feijoeiro (Phaseolus vulgaris L.) vem sendo, ultimamente, preconizado nas várzeas como uma boa alternativa para expansão da fronteira agrícola, incorporando áreas cujo aproveitamento temse limitado quase que exclusivamente ao cultivo do arroz irrigado por inundação contínua. Dadas as limitações desses solos ao uso agrícola, fundamentadas no excesso de água e, ou, baixa fertilidade natural, é imprescindível conhecer sua capacidade de suprir nutrientes às plantas e aprimorar as recomendações de adubação, visando a uma produção eficiente e sustentável ao longo dos anos.

A deficiência de boro (B) nos solos tem provocado grandes perdas de produtividade em algumas culturas brasileiras. Entretanto, a maioria das adubações com o micronutriente são realizadas somente em solos onde já foi constatada sua deficiência, não levando em consideração o teor inicial de B no solo para se fazer uma recomendação correta.

$\mathrm{Na}$ avaliação da disponibilidade de $\mathrm{B}$ no solo, o método da água quente, proposto por Berger \& Truog (1939) parece ainda ser o mais apropriado, embora haja tentativas de se usarem procedimentos mais simples, como a extração com soluções ácidas ou salinas (Ribeiro \& Tucunango Sarabia, 1984; Tedesco et al., 1985). Entretanto, a quantidade de B extraída do solo depende da concentração e da composição química de cada extrator e de atributos químicos, físicos e mineralógicos de cada solo, o que tem conduzido a variações no nível crítico de B no solo. Além disso, a grande heterogeneidade dos solos de várzea, tem mostrado que não se pode generalizar os problemas nutricionais e respostas ao fornecimento de B e outros micronutrientes nesses solos, pois eles são dependentes do tipo de solo e da cultura (Galrão, 1990; Paula et al., 1990).
Essas considerações justificam a necessidade de se dispor de parâmetros adequados para recomendar adubação boratada a diferentes tipos de solos bem como para avaliar o estado nutricional das plantas, considerando também as grandes variações de exigências nutricionais das espécies vegetais.

O objetivo deste trabalho foi estimar os níveis críticos inferiores e superiores de $\mathrm{B}$ em solos de várzea e em plantas de feijoeiro cultivadas em amostras desses solos.

\section{MATERIAL E MÉTODOS}

O feijoeiro (Phaseolus vulgaris L. cv. Carioca) foi cultivado em condições de casa de vegetação do Departamento de Ciência do Solo da Universidade Federal de Lavras (MG), no período de 6 de maio a 16 de agosto de 1997.

Foram utilizadas amostras de quatro solos de várzea nunca cultivados: Aluvial (A), Glei Pouco Húmico (GP), Glei Húmico $(\mathrm{GH})$ e Orgânico $(\mathrm{O})$, este último artificialmente drenado, coletadas da camada superficial $(0-20 \mathrm{~cm})$, em uma única várzea no município de Lavras, $\mathrm{MG}$, secadas, e passadas em peneira com malha de $5 \mathrm{~mm}$ de abertura.

Subamostras foram tomadas para caracterização química e física, avaliando-se o pH em água, $\mathrm{Ca}, \mathrm{Mg}, \mathrm{Al}, \mathrm{P}$ e K (Vettori, 1969, com modificações da Embrapa, 1997); o B disponível, pela água quente (Berger \& Truog, 1939) e determinado no extrato com azometina - H (Malavolta et al., 1997); a acidez potencial (H+Al) e o carbono orgânico (Raij et al., 1987); o Zn, com DTPA (Lindsay \& Norvell, 1978), e a granulometria, pelo método da pipeta (Day, 1965) (Tabela 1).

O experimento foi arranjado num esquema fatorial $4 \times 7$, com quatro repetições, sendo os fatores: quatro solos (GP, A, GH e O) e sete doses de B $(0,0,0,25,0,5,1,5,3,0$, 6,0 e $10,0 \mathrm{mg} \mathrm{dm}^{-3}$ ), em delineamento inteiramente casualizado.

A correção da acidez dos solos, bem como o fornecimento de $\mathrm{Ca}$ e $\mathrm{Mg}$, foram feitos com o uso de calcário dolomítico calcinado, com $35 \%$ de $\mathrm{CaO}, 14 \%$ de $\mathrm{MgO}$ e

TABELA 1. Atributos químicos e físicos das amostras de solos de várzea usados no experimento.

\begin{tabular}{|c|c|c|c|c|c|c|c|c|c|c|c|c|c|}
\hline Solo $^{1}$ & $\mathrm{pH}$ & $\mathrm{P}$ & $\mathrm{K}$ & $\mathrm{B}$ & $\mathrm{Zn}$ & $\mathrm{Ca}$ & $\mathrm{Mg}$ & $\mathrm{Al}$ & $\mathrm{H}+\mathrm{Al}$ & Mat. org & Areia & Silte & Argila \\
\hline & & \multicolumn{4}{|c|}{ - $\left(\mathrm{mg} \mathrm{dm}^{-3}\right)$} & \multicolumn{4}{|c|}{-------( $\left(\mathrm{cmol}_{\mathrm{c}} \mathrm{dm}^{-3}\right)$------- } & \multicolumn{4}{|c|}{ 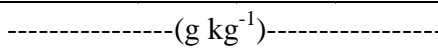 } \\
\hline GP & 5,3 & 3 & 36 & 0,18 & 1,2 & 0,6 & 0,2 & 1,1 & 6,3 & 31 & 641 & 179 & 180 \\
\hline A & 5,2 & 3 & 103 & 0,23 & 2,4 & 2,7 & 1,1 & 0,2 & 4,5 & 31 & 179 & 470 & 351 \\
\hline $\mathrm{GH}$ & 5,4 & 8 & 51 & 0,21 & 2,1 & 0,7 & 0,2 & 1,6 & 13,7 & 230 & 240 & 390 & 370 \\
\hline $\mathrm{O}$ & 5,4 & 6 & 76 & 0,16 & 1,9 & 1,6 & 1,2 & 0,9 & 9,8 & 44 & 120 & 570 & 310 \\
\hline
\end{tabular}

${ }^{1}$ GP: Glei Pouco Húmico; A: Aluvial; GH: Glei Húmico; O: Orgânico. 
$100 \%$ de PRNT, nas doses 13,4, 4,6, 6,4 e 4,6 $\mathrm{t} \mathrm{ha}^{-1}$, para os solos GP, A, GH e O, respectivamente, conforme Andrade (1997).

Os solos receberam como adubação no plantio: $70 \mathrm{mg}$ de $\mathrm{N} ; 300 \mathrm{mg}$ de $\mathrm{P} ; 100 \mathrm{mg}$ de $\mathrm{K} ; 40 \mathrm{mg}$ de $\mathrm{S} ; 1,5 \mathrm{mg}$ de $\mathrm{Cu}$, e 5,0 mg de $\mathrm{Zn}$ por $\mathrm{dm}^{3}$, e as respectivas doses de B, na forma de ácido bórico p.a. $\left(\mathrm{H}_{3} \mathrm{BO}_{3}\right)$. As fontes utilizadas foram sais p.a.: $\mathrm{Ca}\left(\mathrm{H}_{2} \mathrm{PO}_{4}\right)_{2} .2 \mathrm{H}_{2} \mathrm{O} ; \mathrm{KH}_{2} \mathrm{PO}_{4}$; $\mathrm{NH}_{4} \mathrm{H}_{2} \mathrm{PO}_{4} ; \mathrm{CaSO}_{4} .2 \mathrm{H}_{2} \mathrm{O} ; \mathrm{ZnSO}_{4} .7 \mathrm{H}_{2} \mathrm{O} ; \mathrm{CuSO}_{4} .5 \mathrm{H}_{2} \mathrm{O}$; $\left(\mathrm{NH}_{4}\right)_{2} \mathrm{SO}_{4} ; \mathrm{NH}_{4} \mathrm{NO}_{3}$ e $\mathrm{KNO}_{3}$.

Os solos foram incubados em porções de $4 \mathrm{dm}^{3} \mathrm{em}$ sacos de plástico com umidade correspondente a $70 \%$ do volume total de poros ocupados por água durante 24 dias. Após a incubação, $3 \mathrm{dm}^{3}$ de solo foram transferidos para cada vaso, avaliando-se, em amostras do restante, os teores de $\mathrm{B}$ tornados disponíveis pelos extratores $\mathrm{BaCl}_{2}$ 0,125\% (Abreu et al., 1994), Mehlich I (Mehlich, 1978), água quente (Berger \& Truog, 1939) e $\mathrm{CaCl}_{2} 0,01 \mathrm{~mol} \mathrm{~L}^{-1}$ (Tedesco et al., 1985).

Foram conduzidas três plantas de feijoeiro por vaso, mantendo-se a umidade dos solos em torno de $70 \%$ do volume total de poros ocupados por água, por meio de pesagens diárias dos vasos e adição de água deionizada.

Foram realizadas adubações em cobertura com $\mathrm{N} \mathrm{e} \mathrm{K}$, diferenciadas, em função do crescimento das plantas. Os tratamentos que proporcionaram em cada solo um crescimento normal das plantas, receberam $110 \mathrm{mg} \mathrm{dm}^{-3} \mathrm{de} \mathrm{N}^{-}$ e $70 \mathrm{mg} \mathrm{dm}^{-3}$ de $\mathrm{K}$, parcelados em três aplicações. Os tratamentos cujas plantas apresentaram menor crescimento receberam coberturas proporcionalmente menores, evitando-se, assim, aplicações excessivas dos nutrientes.

A parte aérea de uma planta foi colhida no florescimento (estádio R6), e a das outras duas plantas, na maturação fisiológica dos grãos (estádio R9). Todo material vegetal foi secado em estufa de circulação forçada de ar, à tempera- tura de $65-70^{\circ} \mathrm{C}$. Nas plantas colhidas no final do ciclo foi determinado o peso da matéria seca de grãos, e nas colhidas no florescimento foram analisados os teores foliares de B, de acordo com Malavolta et al. (1997).

As variáveis determinadas foram submetidas à análise de variância e de regressão. As equações de regressão foram ajustadas às médias de produção de matéria seca de grãos (MSGR) em vista das doses de B aplicadas. A partir das equações obtidas, estimaram-se as doses de B para se atingir a produção máxima e $90 \%$ da máxima (dose inferior), a dose acima da máxima suficiente para promover uma redução de 10\% na MSGR (dose superior), em cada solo. Substituindo-se essas doses nas equações de regressão que relacionam as doses de $\mathrm{B}$ aplicadas aos solos com seus teores disponíveis pelos extratores $\mathrm{BaCl}_{2} 0,125 \%$, Mehlich I, água quente e $\mathrm{CaCl}_{2} 0,01 \mathrm{~mol} \mathrm{~L}^{-1}$, estimaramse os níveis críticos inferior (para 90\% da MSGR máxima) e superior (para redução de $10 \%$ da MSGR máxima) de B em cada solo. Da mesma maneira, os níveis críticos inferior e superior de B nas folhas do feijoeiro foram estimados pela substituição das doses de B correspondentes, nas equações de regressão que relacionam as doses de B aplicadas aos solos, com seus teores nas folhas do feijoeiro na época do florescimento.

\section{RESULTADOS E DISCUSSÃO}

A análise de variância mostrou que os solos e as doses de $\mathrm{B}$ influenciaram significativamente $(\mathrm{P}<0,01)$ a produção de matéria seca de grãos (MSGR), houve interação entre os fatores. Em todos os solos estudados, a equação quadrática base raiz quadrada foi a que melhor se ajustou para a produção de MSGR, em decorrência das doses de B aplicadas (Tabela 2).

TABELA 2. Equações de regressão ajustadas entre a produção de matéria seca de grãos (MSGR) (Y em g vaso-1), como variável dependente das doses de $B$ aplicadas $\left(X \mathrm{em} \mathrm{mg} \mathrm{dm}^{-3}\right)$ aos solos de várzea e produção estimada de MSGR correspondente à produção máxima e $90 \%$ da máxima e as doses de $B$ estimadas para promover essas produções e redução de $10 \%$ da máxima.

\begin{tabular}{|c|c|c|c|c|c|c|c|}
\hline \multirow[t]{2}{*}{ Solo $^{1}$} & \multirow[t]{2}{*}{ Equação } & \multirow[t]{2}{*}{$\mathrm{R}^{2}$} & \multicolumn{2}{|c|}{ MSGR (g vaso $\left.{ }^{-1}\right)$} & \multicolumn{3}{|c|}{ Dose de B $\left(\mathrm{mg} \mathrm{dm}^{-3}\right)$} \\
\hline & & & $90 \%$ & Máxima & $90 \%$ & Máxima & $-10 \%$ \\
\hline GP & $Y=0,77+9,8715 * * X^{0,5}-2,9563 * * X$ & 0,79 & 8,11 & 9,01 & 1,25 & 2,79 & 4,94 \\
\hline A & $Y=3,58+11,1084 * * X^{0,5}-3,2996 * * X$ & 0,78 & 11,64 & 12,93 & 1,12 & 2,83 & 5,33 \\
\hline $\mathrm{GH}$ & $Y=2,45+17,7416 * * X^{0,5}-5,5891 * * X$ & 0,86 & 14,88 & 16,53 & 1,09 & 2,52 & 4,54 \\
\hline $\mathrm{O}$ & $Y=2,56+13,0703 * * X^{0,5}-4,1346 * * X$ & 0,80 & 11,61 & 12,90 & 1,04 & 2,50 & 4,58 \\
\hline
\end{tabular}

${ }^{1}$ GP: Glei Pouco Húmico; A: Aluvial; GH: Glei Húmico; O: Orgânico.

** Significativo a $1 \%$ de probabilidade. 
O modelo explica aumentos significativos na produção de grãos com a aplicação de pequenas doses de $\mathrm{B}$; e após atingir um ponto máximo, a produção decresce em resposta às doses mais altas de $\mathrm{B}$, o que indica efeito tóxico do micronutriente. A partir dessas equações, estimaram-se as doses de B correspondentes à produção máxima, $90 \%$ da máxima (zona de deficiência) e redução de 10\% da máxima (zona de toxidez) (Tabela 2).

As equações que relacionam o B disponível pelos quatro extratores e as doses do micronutriente aplicadas em cada solo são apresentadas na Tabela 3. A variação dos coeficientes angulares das equações entre os solos para um mesmo extrator é reflexo dos atributos dos solos na capacidade de recuperação do elemento pelo extrator. Entre os atributos do solo, os que exercem maior influência na retenção de B são a CTC, o teor e tipo de argila, os óxidos de $\mathrm{Fe}$ e $\mathrm{Al}$, a matéria orgânica, a superfície específica e o pH (Elrashidi \& O'Connor, 1982). Uma vez que esses atributos apresentam-se em valores bem distintos nos solos estudados (Mariano, 1998), é natural que a disponibilidade do nutriente para as plantas e a quantidade extraída por cada extrator sejam diferentes.
Os níveis críticos inferiores e superiores de B nos solos são apresentados na Tabela 4, os quais mostram ampla variação em cada solo, determinados pelos diferentes extratores. Os níveis críticos inferiores variaram de um valor mínimo de 0,57 a um máximo de $1,87 \mathrm{mg} \mathrm{dm}^{-3}$ de B para os extratores Mehlich I e $\mathrm{CaCl}_{2}$, respectivamente, no solo GH. De modo geral, os níveis críticos inferiores de $\mathrm{B}$ foram maiores com o uso do $\mathrm{CaCl}_{2}$, reflexo do seu maior poder de extração quando comparado aos demais extratores.

O nível crítico inferior de $B$ determinado pela água quente variou entre 0,78 a $1,34 \mathrm{mg} \mathrm{dm}^{-3}$, dependendo do solo estudado. Esses valores estão bem acima da faixa obtida por Buzetti et al. (1990a), 0,19 a $0,23 \mathrm{mg} \mathrm{dm}^{-3} \mathrm{de} \mathrm{B}$, como crítica para atingir $90 \%$ da produção máxima estimada de grãos de soja. Esta comparação, apesar de interessante por se tratar de uma leguminosa, é restrita, uma vez que os níveis críticos foram determinados de maneira diferente e em solos distintos, ressaltando-se, nesse caso, a inconveniência das extrapolações, dada grande heterogeneidade dos solos de várzea.

Conforme as classes de fertilidade sugeridas por Raij et al. (1996), teores de B disponível em água quente maiores que $0,6 \mathrm{mg} \mathrm{dm}^{-3}$ são tidos, no solo, como

TABELA3. Equações de regressão dos teores de B disponível avaliados pelos extratores (Y $\mathrm{em} \mathrm{mg} \mathrm{dm}^{-3}$ ), como variável dependente das doses de $B$ aplicadas $\left(X \mathrm{em} \mathrm{mg} \mathrm{dm}^{-3}\right)$ aos solos de várzea.

\begin{tabular}{|c|c|c|c|}
\hline Solo $^{1}$ & Extrator & Equação & $\mathrm{R}^{2}$ \\
\hline \multirow[t]{4}{*}{$\overline{\text { GP }}$} & $\mathrm{BaCl}_{2}$ & $\mathrm{Y}=0,1101+0,5581 * * \mathrm{X}$ & 0,99 \\
\hline & Mehlich I & $\mathrm{Y}=0,3455+0,5580^{* *} \mathrm{X}$ & 0,99 \\
\hline & Água quente & $\mathrm{Y}=0,1630+0,6113^{* *} \mathrm{X}$ & 0,99 \\
\hline & $\mathrm{CaCl}_{2}$ & $Y=0,4359+0,8537 * * X$ & 0,99 \\
\hline \multirow[t]{4}{*}{ A } & $\mathrm{BaCl}_{2}$ & $\mathrm{Y}=0,0413+0,5700^{* *} \mathrm{X}$ & 0,99 \\
\hline & Mehlich I & $\mathrm{Y}=0,3329+0,4106^{* *} \mathrm{X}$ & 0,99 \\
\hline & Água quente & $\mathrm{Y}=0,1797+0,5355^{* *} \mathrm{X}$ & 0,99 \\
\hline & $\mathrm{CaCl}_{2}$ & $\mathrm{Y}=0,2214+0,8024 * * \mathrm{X}$ & 0,99 \\
\hline \multirow[t]{4}{*}{ GH } & $\mathrm{BaCl}_{2}$ & $\mathrm{Y}=0,3033+0,6164 * * \mathrm{X}$ & 0,99 \\
\hline & Mehlich I & $\mathrm{Y}=0,1508+0,3825 * * \mathrm{X}$ & 0,99 \\
\hline & Água quente & $Y=0,7564+0,5362 * * X$ & 0,99 \\
\hline & $\mathrm{CaCl}_{2}$ & $\mathrm{Y}=1,2121+0,6081 * * \mathrm{X}$ & 0,98 \\
\hline \multirow[t]{4}{*}{$\mathrm{O}$} & $\mathrm{BaCl}_{2}$ & $\mathrm{Y}=0,0409+0,6836 * * \mathrm{X}$ & 0,99 \\
\hline & Mehlich I & $Y=0,2529+0,4637 * * X$ & 0,99 \\
\hline & Água quente & $Y=0,2009+0,6110^{* *} \mathrm{X}$ & 0,99 \\
\hline & $\mathrm{CaCl}_{2}$ & $\mathrm{Y}=0,0892+0,6961 * * \mathrm{X}$ & 0,99 \\
\hline
\end{tabular}

${ }^{1}$ GP: Glei Pouco Húmico; A: Aluvial; GH: Glei Húmico; O: Orgânico.

** Significativo a $1 \%$ de probabilidade. 
altos. Os autores recomendam aplicação de B no solo, para cultivo com feijoeiro, somente quando seu teor (água quente) for inferior a $0,21 \mathrm{mg} \mathrm{dm}^{-3}$.

A variação nos níveis críticos encontrados em diversos trabalhos mostra que, assim como a disponibilidade dos nutrientes está relacionada aos atributos do solo e às condições climáticas, a capacidade das plantas em absorvê-los em uma dada concentração na solução do solo, varia também com a espécie vegetal, vindo a confirmar um fato muito discutido de que a fixação do nível crítico de $\mathrm{B} \mathrm{em} 0,5 \mathrm{mg} \mathrm{dm}^{-3}$, extraído em água quente (Mitchell, 1965), não pode ser generalizado para todas espécies e classes de solos.

Os níveis críticos superiores ou de toxidez, apresentaram, dentro de cada extrator, pequena variação entre os solos, à exceção do solo GH no Mehlich I e do solo $\mathrm{O}$ no $\mathrm{CaCl}_{2}$ 0,01 $\mathrm{mol} \mathrm{L}^{-1}$, com valores mais baixos que os demais. Observa-se que na média dos solos, tal como ocorreu para os níveis críticos inferiores, $\mathrm{o} \mathrm{CaCl}_{2} 0,01 \mathrm{~mol} \mathrm{~L}^{-1}$ foi o extrator que apresen- tou os maiores valores para os níveis tóxicos de B, ao passo que os demais apresentaram valores semelhantes e também acima da faixa citada como ótima (0,5 a 2,0 $\mathrm{mg} \mathrm{dm}^{-3} \mathrm{de}$ B) para o feijoeiro (Oliveira \& Thung, 1988). Lindsay \& Cox (1985) citam como níveis tóxicos os que se acham acima de $3,0 \mathrm{mg} \mathrm{dm}^{-3}$ de B. Certamente, as diferenças entre os valores observados nos níveis tóxicos e os citados na literatura, se devem aos tipos de solos, às culturas, aos extratores utilizados, e aos critérios adotados para o estabelecimento de tais valores.

As informações a respeito de níveis tóxicos de $\mathrm{B}$ no solo são escassas, mesmo porque os teores de B disponível encontrados naturalmente nos solos brasileiros são baixos. A toxicidade é mais comum em regiões áridas ou semi-áridas, ou ainda nas relacionadas com água de irrigação com altos teores de B (Marschner, 1995).

As concentrações de B no tecido foliar do feijoeiro no florescimento aumentaram linearmente com as doses aplicadas (Tabela 5). Os coeficientes lineares

TABELA 4. Níveis críticos de B estimados nos solos de várzea para o feijoeiro, correspondentes a $90 \%$ da produção máxima (inferior) e à redução de $10 \%$ da máxima (superior), pelos extratores $\mathbf{B a C l}_{2}$, Mehlich I, água quente e $\mathrm{CaCl}_{2}$.

\begin{tabular}{|c|c|c|c|c|c|c|c|c|}
\hline \multirow[t]{2}{*}{ Solo $^{1}$} & \multicolumn{2}{|c|}{$\mathrm{BaCl}_{2}$} & \multicolumn{2}{|c|}{ Mehlich I } & \multicolumn{2}{|c|}{ Água quente } & \multicolumn{2}{|c|}{$\mathrm{CaCl}_{2}$} \\
\hline & Inferior & Superior & Inferior & Superior & Inferior & Superior & Inferior & Superior \\
\hline & ----------. & ----------- & ------- & - $(n$ & -3) ------ & 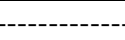 & -----------. & ----------- \\
\hline GP & 0,81 & 2,87 & 1,04 & 3,10 & 0,93 & 3,18 & 1,50 & 4,65 \\
\hline A & 0,68 & 3,08 & 0,79 & 2,52 & 0,78 & 3,03 & 1,12 & 4,50 \\
\hline $\mathrm{GH}$ & 0,98 & 3,10 & 0,57 & 1,89 & 1,34 & 3,19 & 1,87 & 3,97 \\
\hline $\mathrm{O}$ & 0,75 & 3,17 & 0,74 & 2,38 & 0,84 & 3,00 & 0,81 & 3,28 \\
\hline
\end{tabular}

${ }^{1}$ GP: Glei Pouco Húmico; A: Aluvial; GH: Glei Húmico; O: Orgânico.

TABELA 5. Equações de regressão ajustadas dos teores foliares de B (Y em mg kg-1) na época do florescimento, como variável dependente das doses de $B$ aplicadas $\left(X \mathrm{em} \mathrm{mg} \mathrm{dm}{ }^{-3}\right.$ ) e níveis críticos foliares, inferior (para 90\% da MSGR máxima) e superior (para redução de 10\% da MSGR máxima).

\begin{tabular}{lcccc}
\hline Solo $^{1}$ & Equação & $\mathrm{R}^{2}$ & \multicolumn{2}{c}{ Níveis críticos $\left(\mathrm{mg} \mathrm{kg}^{-1}\right)$} \\
\cline { 3 - 5 } & & & Inferior & Superior \\
\hline GP & $\mathrm{Y}=10,4927+26,9473 * * \mathrm{X}$ & 0,99 & 44,2 & 143,6 \\
$\mathrm{~A}$ & $\mathrm{Y}=36,4777+28,2212^{* * \mathrm{X}}$ & 0,99 & 68,1 & 186,9 \\
$\mathrm{GH}$ & $\mathrm{Y}=20,5581+27,1616^{* *} \mathrm{X}$ & 0,99 & 50,2 & 143,9 \\
$\mathrm{O}$ & $\mathrm{Y}=10,7893+41,1232 * * \mathrm{X}$ & 0,98 & 53,6 & 199,1 \\
\hline
\end{tabular}

${ }^{1}$ GP: Glei Pouco Húmico; A: Aluvial; GH: Glei Húmico; O: Orgânico.

** Significativo a $1 \%$ de probabilidade. 
das equações, que indicam o teor foliar de B na dose zero, mostram capacidade bastante diferenciada dos solos em fornecer $\mathrm{B}$ às plantas. Os solos GP e O foram os que proporcionaram menores teores de $\mathrm{B}$ no tecido foliar, o que confirma, mais uma vez, a baixa disponibilidade original de $\mathrm{B}$ nesses solos (Tabela 1). Os acréscimos estimados nos teores foliares de B para cada unidade de B aplicada, mostrados pelos coeficientes angulares das equações, evidenciam magnitude semelhante desses aumentos nos solos GP, A e GH, e magnitude superior aos demais no solo $\mathrm{O}$.

A Tabela 5 mostra que os níveis críticos foliares inferior e superior de B variaram entre os solos, mostrando-se dependentes de alguns de seus atributos, os quais influenciaram, além da disponibilidade de $\mathrm{B}$, também o crescimento das plantas, uma vez que a produção de MSGR estimada para $90 \%$ ou redução de $10 \%$ da máxima, também foi diferente entre os solos. Pode-se inferir, portanto, que os atributos dos solos que influenciam o crescimento das plantas afetam também o coeficiente de utilização do micronutriente, conferindo diferentes valores de níveis críticos na planta. Um fato que comprova claramente essa afirmação pode ser observado nos solos A e $\mathrm{O}$, onde as plantas apresentaram produções semelhantes (Tabela 2), porém os níveis críticos inferior e superior foram diferentes (Tabela 5).

Além desse aspecto, pode-se destacar também que o solo GP foi o que apresentou as menores produções de MSGR (correspondentes a 90\% e redução de $10 \%$ da máxima) e exigiu, em valores absolutos, as maiores doses de B para atingir essas produções (Tabela 2). Por outro lado, neste solo, as plantas apresentaram o menor nível crítico inferior e, juntamente com o GH, o menor nível crítico superior de B foliar, entre os solos (Tabela 5).

Quanto aos níveis críticos inferiores, Paula (1995) encontrou valores entre 17 e $35 \mathrm{mg} \mathrm{kg}^{-1}$ de B na parte aérea do arroz durante o florescimento, cultivado em solos de várzea sob inundação. A autora menciona que o nível de toxicidade deve estar acima de $51 \mathrm{mg} \mathrm{kg}^{-1} \mathrm{de} \mathrm{B}$, a maior concentração de B no tecido foliar obtida com a aplicação de 2,0 mg de B por $\mathrm{dm}^{3}$ de solo, sem a observação de sintomas de toxidez, embora a produção de matéria seca tenha sofrido redução com esta dose. Buzetti et al. (1990b) deter- minaram valores na faixa de 42 a $56 \mathrm{mg} \mathrm{kg}^{-1}$ de B na parte aérea da soja 'Paraná' como críticos para obtenção de $90 \%$ do máximo produzido.

Malavolta et al. (1997) citam como teores foliares adequados para o feijoeiro, no início do florescimento, variando na faixa de 30 a $60 \mathrm{mg} \mathrm{kg}^{-1}$ de B, ao passo que Raij et al. (1996) estabelecem, para todas as folhas amostradas no florescimento, a faixa de 15 a $26 \mathrm{mg} \mathrm{kg}^{-1}$ de B.

Marschner (1995) menciona que as espécies de plantas diferem caracteristicamente quanto ao requerimento de B para o crescimento, e cita, como exemplo, que a faixa crítica de deficiência varia de 5 a $10 \mathrm{mg} \mathrm{kg}^{-1}$ de B em gramíneas, 20 a $70 \mathrm{mg} \mathrm{kg}^{-1}$ de B na maioria das dicotiledôneas, e 80 a $100 \mathrm{mg} \mathrm{kg}^{-1}$ de B em espécies mais exigentes, como a papoula. $\mathrm{O}$ maior requerimento de $\mathrm{B}$ em dicotiledôneas, comparado às gramíneas, está presumidamente relacionado a maiores proporções de compostos cis-diol na parede celular do primeiro grupo, onde grande proporção do conteúdo total de B fica complexado (Loomis \& Durst, 1992). Esta diferença torna-se mais clara quando se observa que os níveis críticos no feijoeiro, neste trabalho, variaram na faixa de 44,2 a $68,1 \mathrm{mg} \mathrm{kg}^{-1}$ de $\mathrm{B}$, ao passo que na cultura do arroz, também em solos de várzea, os valores ficaram entre 17 e $35 \mathrm{mg} \mathrm{kg}^{-1}$ de B (Paula, 1995).

Assim como as espécies diferem no requerimento pelo B, elas o fazem também na tolerância ao elemento. Como exemplo, algumas faixas de teores críticos tóxicos de B no tecido foliar, expressos em mg kg-1, são citadas por Marschner (1995): milho, 100; pepino, 400; abóbora, 1.000; trigo, 100 a 270; feijão, 100; e caupi, $>330$.

As diferenças entre os níveis críticos estimados e os encontrados na literatura, são resultantes, talvez, de fatores como: época de cultivo, método utilizado para determinação desses níveis críticos, doses dos outros nutrientes aplicados, idade da planta ou do órgão amostrado, época de amostragem, e condições de cultivo, entre outros.

A grande variabilidade de atributos dos solos de várzea, com todas as implicações na dinâmica de nutrientes e no desenvolvimento de plantas, ressalta a necessidade de se considerar individualmente o potencial produtivo do solo para cada cultura, a fim de que se utilizem da melhor forma esses sistemas. 


\section{CONCLUSÕES}

1. Nos solos de várzea, os níveis críticos inferiores e superiores de B para o feijoeiro variam, independentemente do extrator, de 0,57 a $1,87 \mathrm{mg} \mathrm{dm}^{-3} \mathrm{e}$ de 1,89 a $4,65 \mathrm{mg} \mathrm{dm}^{-3}$, respectivamente.

2. Nas folhas do feijoeiro, os níveis críticos inferiores e superiores de B variam, respectivamente, de 44,2 a $68,1 \mathrm{mg} \mathrm{kg}^{-1}$ e de 143,6 a 199,1 $\mathrm{mg} \mathrm{kg}^{-1}$.

\section{REFERÊNCIAS}

ABREU, C.A.; ABREU, M.F. de; RAIJ, B. van.; BATAGLIA, O.C.; ANDRADE, J.C. Extraction of boron from soil by microwave heating for ICP-AES determinations. Communications in Soil Science and Plant Analysis, New York, v.25, n.19/20, p.3321-3333, Dec. 1994.

ANDRADE, C.A. de B. Limitações de fertilidade e efeito do calcário para o feijoeiro (Phaseolus vulgaris $\mathbf{L}$.) em solos de várzea do sul de Minas Gerais. Lavras : UFLA, 1997. 107p. Tese de Doutorado.

BERGER, K.C.; TRUOG, E. Boron determination in soils and plants. Industrial and Engineering Chemistry, Washington, v.11, p.540-545, 1939.

BUZETTI, S.; MURAOKA, T.; SÁ, M.E. de. Doses de boro na soja, em diferentes condições de acidez do solo. I. Produção de matéria seca e de grãos e nível crítico no solo. Revista Brasileira de Ciência do Solo, Campinas, v.14, n.2, p.157-161, maio/ago. 1990a.

BUZETTI, S.; MURAOKA, T.; SÁ, M.E. de. Doses de boro na soja, em diferentes condições de acidez do solo. II. Níveis críticos na planta e nos grãos. Revista Brasileira de Ciência do Solo, Campinas, v.14, n.2, p.163-166, maio/ago. 1990b.

DAY, P.R. Particle fractionation and particle-size analysis. In: BLACK, C.A. (Ed.). Methods of soil analysis: physical and mineralogical properties, including statistics of measurement and sampling. Madison : American Society of Agronomy, 1965. p.545-566.

ELRASHIDI, M.A.; O'CONNOR, G.A. Boron sorption and desorption in soils. Soil Science Society of America. Journal, Madison, v.46, n.1, p.27-31, 1982.
EMBRAPA. Centro Nacional de Pesquisa de Solos (Rio de Janeiro, RJ). Manual de métodos de análises de solos. 2.ed. Rio de Janeiro, 1997. 212p. (Documentos 1).

GALRÃO, E.Z. Aplicação de micronutrientes e calcário no rendimento da soja em solo de várzea. Revista Brasileira de Ciência do Solo, Campinas, v.14, n.3, p.381-384, set./dez. 1990.

LINDSAY, W.L.; COX, F.R. Micronutrients soil testing for the tropics. In: VLEK, D.L.G. (Ed.). Micronutrients in tropical food crop production: developments in plant and soil sciences. Dordrecht : M. Vighoff, 1985. v.14, p.169-200.

LINDSAY, W.L.; NORVELL, W.A. Development of a DTPA soil test for zinc, iron, manganese and cooper. Soil Science Society of American. Journal, Madison, v.42, n.3, p.421-428, May/June 1978.

LOOMIS, W.D.; DURST, R.W. Chemistry and biology of boron. Biofactors, Amsterdam, v.3, p.229-239, 1992.

MALAVOLTA, E.; VITTI, G.C.; OLIVEIRA, S.A. Avaliação do estado nutricional das plantas: princípios e aplicações. 2.ed. Piracicaba : Potafos, 1997.319p.

MARIANO, E.D. Respostas, níveis críticos e eficiência de extratores para boro em feijoeiro cultivado em solos de várzea. Lavras : UFLA, 1998. 82p. Dissertação de Mestrado.

MARSCHNER, H. Mineral nutrition of higher plants. San Diego : Academic, 1995. 889p.

MEHLICH, A. New extractant for soil test evaluation of phosphorus, potassium, magnesium, calcium, sodium, manganese and zinc. Communications in Soil Science and Plant Analysis, New York, v.9, p.477-492, 1978.

MITCHELL, R.L. Trace elements in soil In: BEAR, F.E. (Ed.). Chemistry of the soil. New York : Reinhold, 1965. p.320-368

OLIVEIRA, S.A. de; THUNG, M.R.T. Nutrição mineral. In: ZIMMERANN, M.J.O.; ROCHA, M.; YAMADA, T. (Ed.). Cultura do feijoeiro: fatores que afetam a produtividade. Piracicaba : Potafos, 1988. p.175-212.

PAULA, M.B. de. Eficiência de extratores e níveis críticos de boro disponível em amostras de solos aluviais e hidromórficos sob a cultura do arroz

Pesq. agropec. bras., Brasília, v.35, n.8, p.1637-1644, ago. 2000 
inundado. Lavras : UFLA, 1995. 69p. Tese de Doutorado.

PAULA, M.B. de; CARVALHO, J.G. de; SOARES, A.A.; NOGUEIRA, F.D. Avaliação da fertilidade de um solo de várzea (Glei Húmico) para a cultura do arroz. Pesquisa Agropecuária Brasileira, Brasília, v.25, n.4, p.571-577, abr. 1990.

RAIJ, B. van.; CANTARELLA, H.; QUAGGIO, J.A.; FURLANI, A.N.C. (Ed.). Recomendações de adubação e calagem para o Estado de São Paulo. Campinas : Instituto Agronômico, 1996. 285p. (IAC. Boletim Técnico, 100).

RAIJ, B. van.; QUAGGIO, J.A.; CANTARELLA, H.; FERREIRA, M.E.; LOPES, A.S.; BATAGLIA, O.C.
Análise química do solo para fins de fertilidade. Campinas : Fundação Cargill, 1987. 170p.

RIBEIRO, A.C.; TUCUNANGO SARABIA, W.A.T. Avaliação de extratores para zinco e B disponíveis em Latossolos do Triângulo Mineiro. Revista Brasileira de Ciência do Solo, Campinas, v.8, n.1, p.85-89, jan./abr. 1984.

TEDESCO, M.J.; VOLKWEISS, S.J.; BOHNEN, H. Análise de solo, plantas e outros materiais. Porto Alegre : UFRGS, 1985. 188p. (Boletim Técnico, 5).

VETTORI, L. Métodos de análise de solo. Rio de Janeiro : Ministério da Agricultura, 1969. 24p. (Boletim Técnico, 7). 